\title{
Destress 9-1-1-an online mindfulness-based intervention in reducing stress among emergency medical dispatchers: a randomised controlled trial
}

\author{
Michelle Lilly, ${ }^{1}$ Rebecca Calhoun, ${ }^{2}$ Ian Painter, ${ }^{2}$ Randal Beaton, ${ }^{2}$ Scott Stangenes, ${ }^{2}$ \\ Debra Revere, ${ }^{2}$ Janet Baseman, ${ }^{2}$ Hendrika Meischke ${ }^{2}$
}

- Additional material is published online only. To view please visit the journal online (http://dx.doi.org/10.1136/ oemed-2018-105598).

${ }^{1}$ Psychology, Northern Illinois University, DeKalb, Illinois, USA ${ }^{2}$ Northwest Center for Public Health Practice, University of Washington, DeKalb, Illinois, USA

\section{Correspondence to} Dr Michelle Lilly, Psychology, Northern Illinois University, DeKalb, IL 60115, USA: mlilly1@niu.edu

Received 20 November 2018 Revised 1 May 2019 Accepted 6 May 2019 Published Online First 28 May 2019

\begin{abstract}
Objectives Emergency medical dispatchers (EMDs) experience significant stress in the workplace. Yet, interventions aimed at reducing work-related stress are difficult to implement due to the logistic challenges associated with the relatively unique EMD work environment. This investigation tested the efficacy of a 7-week online mindfulness-based intervention (MBI) tailored to the EMD workforce.

Methods Active-duty EMDs from the USA and Canada $(n=323)$ were randomly assigned to an intervention or wait list control condition. Participants completed surveys of stress and mindfulness at baseline, post intervention, and 3 months follow-up. Repeated measures mixed effects models were used to assess changes in stress and mindfulness.
\end{abstract}

Results Differences between the intervention group and control group in pre-post changes in stress using the Calgary Symptoms of Stress Inventory were statistically significant, with a difference of $-10.0(95 \%$ $\mathrm{Cl}:-14.9,-5.2, \mathrm{p}<0.001)$ for change from baseline to post intervention, and a difference of $-6.5(95 \%$ $\mathrm{Cl}:-11.9,-1.1, \mathrm{p}=0.02)$ for change from baseline to 3 months follow-up. Change in mindfulness scores did not differ between groups. However, increases in mindfulness scores were correlated with greater reductions in stress for all participants, regardless of group $(r=-0.53, p<0.001)$.

Conclusions Development of tailored online MBIs for employees working in challenging work environments offer a promising direction for prevention and intervention. This study found that a short, weekly online $\mathrm{MBI}$ for EMDs resulted in reductions in reports of stress. Implications of online MBIs in other emergency responding populations and directions for future research are discussed.

\section{INTRODUCTION}

Emergency responders are repeatedly exposed to duty-related events of a stressful nature. Occupational stress is reportedly high among emergency responders, ${ }^{12}$ which may partially account for the higher prevalence of psychopathology ${ }^{34}$ and physical health symptoms and disease ${ }^{56}$ among this population when compared with the general public. Yet, much of this literature has relied on field emergency responding populations such as police and firefighters.

\section{Key messages}

What is already known about this subject?

- Research has shown mindfulness-based interventions (MBIs), including online MBIs, to be effective in reducing stress among varied workplace populations.

What are the new findings?

- A 7-week MBI presented online significantly reduced reported levels of stress among emergency medical dispatchers when compared with a control group.

- Reductions in stress were observed from pretreatment to post-treatment, as well as from pretreatment to a 3-month follow-up.

\section{How might this impact on policy or clinical practice in the foreseeable future? \\ - Online MBIs are a promising direction for clinical prevention and intervention, as this approach offers affordability, convenience and greater geographical reach. \\ - Demonstration that such an approach can significantly reduce stress in an emergency responding population indicates that similar populations may benefit in the future from such tailored interventions, with implications for public health.}

As the first of the first responders, emergency medical dispatchers (EMDs) are repeatedly exposed to stressful duty-related events. They may provide life-saving instructions over the phone while simultaneously dispatching field responders, or they may be the last individual to speak with an injured individual who, despite their best efforts, they were unable to save. The EMD work environment is typically marked by shift work schedules, mandatory overtime, rare and unpredictable breaks and an inability to ambulate while on duty. Research on the health and wellness of this population is limited. Among EMDs in the Pacific Northwest, the highest self-reported symptoms of stress were sympathetic arousal, muscle tension and anger. ${ }^{7}$

One factor that may be targeted for change among EMDs is mindfulness. Mindfulness is defined as awareness that arises through paying attention, on purpose, in the present moment, 
non-judgementally. ${ }^{8}$ Low levels of mindfulness have been connected with greater reports of stress and poor mental health outcomes across a variety of populations. ${ }^{9}{ }^{10}$ Research on stress and mindfulness in emergency responders is limited. In a longitudinal study of police recruits, researchers found that lower levels of baseline mindfulness predicted future depression symptoms after 1 year, ${ }^{11}$ and greater mindfulness has been associated with lower overall stress among EMDs. ${ }^{7}$

A growing research base supports the use of mindfulness-based interventions (MBIs) to decrease stress in workplace populations. Mindfulness can be cultivated through repeated practice of exercises that improve contact with the present moment (eg, focused breathing and mindful eating). In a recent meta-analysis of studies instituting MBIs to reduce stress in the workplace $(n=19)$, Virgili found medium-to-large effect sizes from pre to post MBI (Hedges's g=0.68). ${ }^{12}$ Huang, Li, Huang, and Tang randomised workers into an MBI or control group and found reduced general distress and perceived stress in an MBI group in comparison to a control group. ${ }^{13}$ In regard to emergency responders, one study found MBI to be associated with significant improvements in perceived stress, global mental health and global physical health. ${ }^{14}$ However, the study was limited by the small sample size $(n=43)$ and lack of a control group. Bergman et al found decreases in organisational stress, operational stress and anger following MBI in another study of police officers using a within-subjects design. ${ }^{15}$

This randomised controlled trial was designed to evaluate the efficacy of an online mindfulness training in reducing stress in EMDs. A recent meta-analysis revealed that online MBIs show significant positive effects on stress. ${ }^{16}$ Further, guided online MBIs, such as the present intervention, were associated with larger effects than unguided $\mathrm{MBIs}(\mathrm{Q}=20.12$, $\mathrm{p}<0.001)$. It was anticipated that EMDs randomly assigned to the online MBI would report a greater reduction in stress and greater improvement in mindfulness scores post intervention and at 3 months follow-up compared with EMDs assigned to the control group.

\section{METHOD}

\section{Participants}

Participants included 323 active-duty 9-1-1 telecommunicators (ie, EMDs) from across the USA and Canada. Most participants were between 26 and 35 years of age $(33.6 \% ; n=108)$ or 36 and 45 years of age $(33.0 \% ; n=106)$. Women $(81.9 \% ; n=262)$ and European Americans $(71.3 \% ; n=299)$ comprised the majority of the sample, and most participants had served as an EMD for at least 2 years $(>70 \%)$.

\section{Measures}

\section{Stress symptoms}

The Calgary Symptoms of Stress Inventory (C-SOSI) is a 56-item measure used to assess stress symptoms over the past week, the primary outcome variable for this study. ${ }^{17}$ The C-SOSI was completed at baseline (T1), post intervention (T2) and 3 months follow-up (T3). Participants respond to items using a 5-point Likert scale ranging from ' 0 (never)' to ' 4 (very frequently)'. The C-SOSI score is the sum of all responses with possible values ranging from 0 to 224. The C-SOSI has been used in previous research with $\mathrm{EMDs}^{7}$ and in a recent intervention study targeting stress. ${ }^{18}$ Internal consistency for the C-SOSI score in the current study was $\alpha=0.953$.
Mindfulness

The Mindfulness Scale (The Mindful Attention Awareness Scale (MAAS)) assessed mindfulness conceptualised as attentional sensitivity to psychological, somatic and environmental cues. ${ }^{19}$ The MAAS was the secondary outcome of interest and was completed at T1, T2 and T3. Response options are on a 6-point scale ranging from 'Almost always' to 'Almost never'. Initial psychometric properties of the MAAS were examined in college students and in adults from the general population. ${ }^{19}$ The MAAS has been employed in previous research with EMDs, ${ }^{7}$ as well as recent intervention studies targeting mindfulness. ${ }^{20} 21$ Internal consistency for the MAAS score in the current study was $\alpha=0.912$.

\section{Demographic information}

Participants reported at baseline their age, gender, ethnicity and years of experience in the EMD field.

\section{Procedure}

Participants were recruited from enroled 9-1-1 call centres across the USA and Canada. All active-duty EMDs were eligible to participate with no exclusionary criteria. Call centre administrators sent emails to employees describing the research and providing contact information for the research team. Interested individuals contacted the research team and were provided a link to the baseline questionnaire. Participants were recruited to complete the baseline survey on a rolling basis over an 18-month period spanning 2016 and 2017. Informed consent was provided electronically prior to completing the baseline survey. Following the baseline survey, participants were randomly assigned to either the intervention or wait-list control group using a random number generator completed prior to participant enrolment. Participants in the intervention condition were asked to begin the intervention within 1 week. Following the intervention or a 7-week wait period for control participants, a post-treatment survey was completed (T2). A follow-up survey was administered 3 months following the post-intervention survey (T3). Participants were debriefed online at the conclusion of each survey time point. Participants in the control group were given access to the online intervention after completion of the T3 survey. The full study protocol was approved by the Institutional Review Board at the University of Washington. The trial was not preregistered. A protocol paper describing the study and intervention in greater detail is published in Open Access. ${ }^{22}$

\section{Intervention}

Greater detail regarding the intervention content, themes and practice activities is available elsewhere. ${ }^{22}$ The online intervention (Destress 9-1-1) comprised seven modules each completed on a weekly basis. Two emails were sent each week: one introducing the weekly theme and one providing practice reminders. Completion times for each module ranged from 20 to $30 \mathrm{~min}$ and included a short video introducing the weekly theme, text describing themes and activities, an audio-guided meditation exercise, suggestions for daily mindfulness activities and a moderated discussion board. Audio-guided exercises were recorded by the second author. Exercises were largely meditation-based or designed to enhance mindfulness during daily activities (ie, mindful movement). The online intervention was developed by clinicians trained in mindfulness-based approaches. The training was informed by Mindfulness-Based Stress Reduction (MBSR), which is an evidence-based programme originally implemented in-person ${ }^{8} 23$; however, the adapted intervention in this study 
differs from formal MBSR in length, format (online) and expectations for outside practice (5-10 min daily vs $20-45 \mathrm{~min}$ daily in MBSR).

Participants were asked at each weekly check-in the number of days that they practised mindfulness using the guided audio and whether the participant incorporated mindfulness into their daily life. In addition, the online training system tracked participants' progress through each lesson, allowing the researchers to accurately assess the number of lessons completed by each participant. No unintended or adverse outcomes were recorded.

\section{Statistical analysis}

Following the previously published protocol, ${ }^{22}$ the primary analyses used repeated measures mixed effects models, with differences assessed by interaction terms between randomisation group and time point. All participants who completed at least one survey were included in analyses. Because differential follow-up rates between study groups were anticipated, a sensitivity analysis to determine the level of difference in response between completers and non-completers needed to change the analysis results at T2 was conducted using multiple imputation. A multivariable model was developed predicting T2 scores using T1 measures associated with post-training score or post-training non-response. All variables with statistically significant associations were included in the imputation model. Time 2 scores for participants missing T2 data were then imputed using this model with an additional bias term inflating post-training SOSI scores by a fixed amount that systematically varied across sets of simulations. The level of bias required to produce no difference in SOSI scores between the groups and level of bias required to achieve a non-statistically significant association between training and SOSI scores in $20 \%$ of the simulations were estimated. Because the training was offered to participants in the control group after T3, it was possible to identify participants in the control group who subsequently completed the training. This allows for a direct comparison of the effects of completing the training on change in stress from T1 to T2 between participants who completed the training (completers) in the intervention group with completers in the control group. An ad-hoc subgroup analysis was conducted using a two-sample t-test to compare change in C-SOSI scores from time T1 to T2 between these two groups. An $\alpha$ level of 0.05 was used for all analyses. All statistical analyses were conducted in R V.3.3.1. ${ }^{24}$

\section{RESULTS}

Figure 1 provides a CONSORT flow diagram of participant retention. Demographic information for each group can be seen in table 1. Although education differed across groups, it was not related to change in stress scores and therefore not included as a control variable in subsequent analyses. Race was associated with survey non-completion at T2 (table 2). However, race was neither associated with survey completion at T3 nor changes in stress score, and thus, was not included in subsequent analyses.

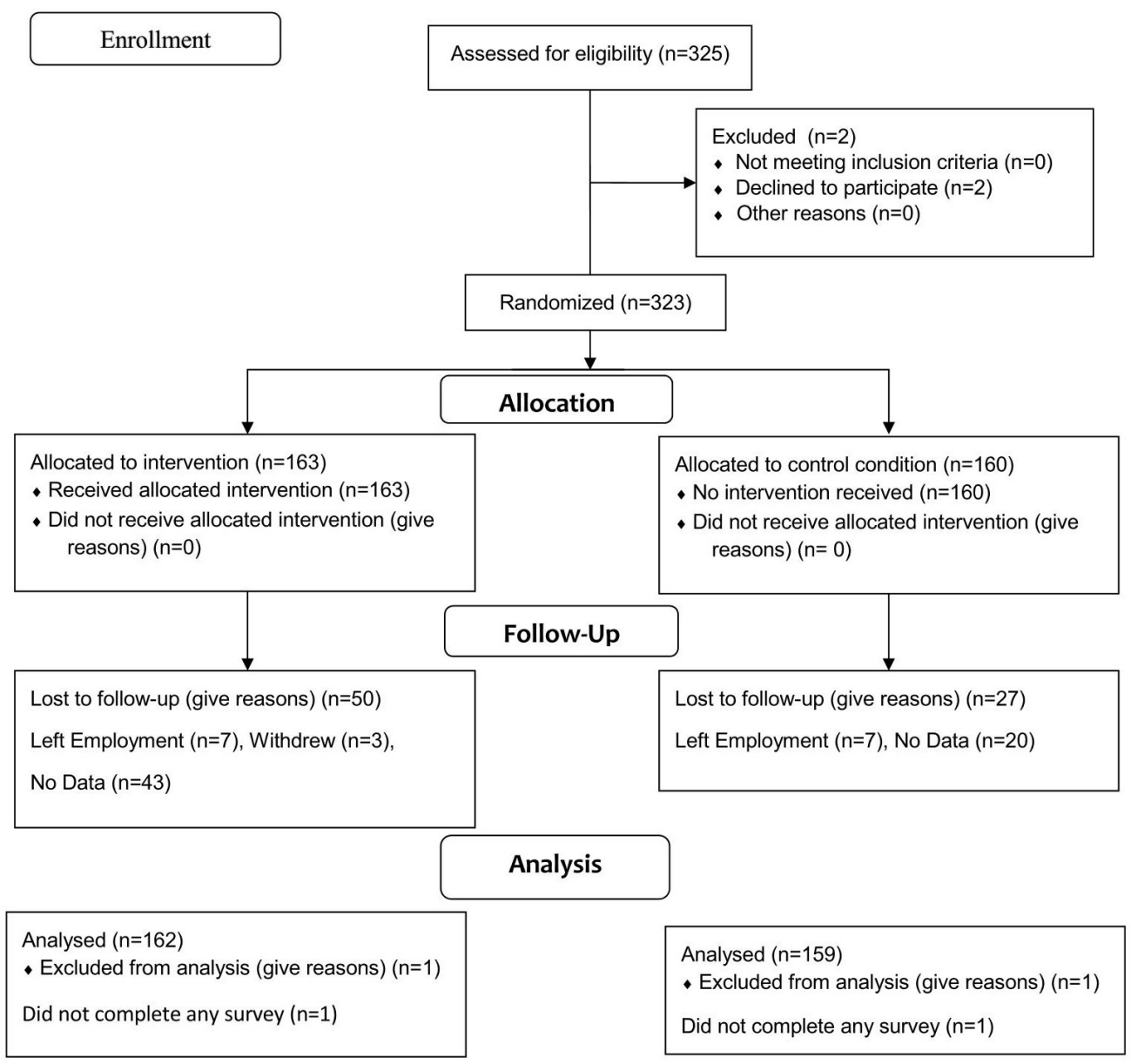

Figure 1 CONSORT flow diagram of participant enrolment and retention. CONSORT, Consolidated Standards of Reporting Trials. 
Table 1 Demographic characteristics of participants by group

\begin{tabular}{|c|c|c|c|c|c|}
\hline & \multicolumn{2}{|c|}{ Intervention group } & \multicolumn{2}{|c|}{$\begin{array}{l}\text { Control } \\
\text { group }\end{array}$} & \multirow[b]{2}{*}{$P$ value } \\
\hline & $\mathrm{N}$ & $\%$ & $\mathrm{~N}$ & $\%$ & \\
\hline \multicolumn{6}{|l|}{ Age } \\
\hline$<25$ years & 19 & 11.9 & 12 & 7.4 & 0.68 \\
\hline $25-35$ years & 55 & 34.4 & 54 & 33.1 & \\
\hline $36-45$ years & 50 & 31.2 & 57 & 35.0 & \\
\hline $46-55$ years & 28 & 17.5 & 30 & 18.4 & \\
\hline 56-64 years & 8 & 5.0 & 10 & 6.1 & \\
\hline \multicolumn{6}{|l|}{ Years worked as EMD } \\
\hline$<2$ years & 22 & 13.8 & 33 & 13.6 & 1.00 \\
\hline $2-5$ years & 37 & 23.1 & 38 & 23.5 & \\
\hline $6-10$ years & 33 & 20.6 & 35 & 21.6 & \\
\hline $11-20$ years & 50 & 31.2 & 48 & 29.6 & \\
\hline $21-30$ years & 13 & 8.1 & 14 & 8.6 & \\
\hline \multicolumn{6}{|l|}{ Education } \\
\hline High school/GED & 29 & 18.2 & 15 & 9.2 & 0.043 \\
\hline Some college & 76 & 47.8 & 69 & 42.3 & \\
\hline Associates/bachelors degree & 49 & 30.8 & 60 & 42.9 & \\
\hline Post-graduate study or degree & 5 & 3.1 & 9 & 5.5 & \\
\hline \multicolumn{6}{|l|}{ Gender } \\
\hline Male & 32 & 20.1 & 26 & 16 & 0.385 \\
\hline Female & 127 & 79.9 & 137 & 84 & \\
\hline \multicolumn{6}{|l|}{ Race } \\
\hline Non-White & 14 & 8.8 & 17 & 10.5 & 0.53 \\
\hline White & 146 & 91.2 & 145 & 89.5 & \\
\hline \multicolumn{6}{|l|}{ Ethnicity } \\
\hline Hispanic & 2 & 1.3 & 4 & 2.5 & 0.685 \\
\hline Non-Hispanic & 157 & 98.7 & 156 & 97.5 & \\
\hline
\end{tabular}

EMD, emergency medical dispatchers.

\section{Attrition}

Survey non-completion differed significantly between the intervention and control groups at T2 and T3. At T2, 30 control participants (18.8\%) and 51 intervention participants $(31.7 \%)$ failed to complete the survey. At T3, 60 control participants $(37.5 \%)$ and 75 intervention participants (46.6\%) failed to complete the survey. Survey non-completion at T2 $(\mathrm{p}=0.26)$ and T3 $(p=0.13)$ was not associated with baseline stress for either group. Level of participation in the training was associated with survey completion at T2 and T3 for the intervention group, with $78 \%$ non-response for participants who completed no trainings, $38 \%$ for participants who completed 1 to 5 trainings and $9 \%$ for participants who completed 6 or 7 of the trainings $(\mathrm{p}<0.001)$.

\section{Intervention dosage}

Among intervention participants, 40 (24.8\%) completed no sessions, 32 (19.9\%) completed 1 to 5 sessions and 89 (55.3\%) completed 6 or 7 sessions. Mean number of days per week on which mindfulness was practised was $2.1(\mathrm{SD}=1.5$, range $0-6.8)$. No statistically significant association was observed between number of training sessions completed and number of days on which mindfulness was practised for participants who completed at least one session.

\section{Intervention effects}

Mean C-SOSI scores increased from T1 to T2 in the control group by 3.1 points and decreased by 7.2 points in the intervention group (table 3 ). The difference in pre--post change between groups was statistically significant, with the change being 10.0 points lower (95\% CI: 5.2, 14.9, p<0.001) in the intervention group at T2, and 6.5 points lower $(95 \% \mathrm{CI}: 1.1,11.9, \mathrm{p}=0.030$ ) at T3.

To control for the possibility that participants' willingness to complete the training, rather than training effects, was associated with change in stress symptoms, an ad-hoc subgroup analysis was conducted comparing participants who completed the training in the intervention group $(n=88)$ with participants in the control group who subsequently completed the training when offered after T3 $(n=38)$. A difference of -8.1 (95\% CI: $-15.1,-0.2, p=0.025)$ for change from T1 to T2 was observed, and a difference of -6.7 (95\% CI: $-15.4,2.0, \mathrm{p}=0.131)$ was observed for change from T1 to T3 (table 4).

\section{Sensitivity analyses}

To negate the statistically significant association between group and change in stress at T2, participants with no post-training C-SOSI scores would need to have mean post-training SOSI scores eight points higher than that predicted by the imputation model. To produce no difference in SOSI scores would require mean post-training SOSI scores to be on average 50 points higher than predicted by the imputation model for participants who did not complete the post-training survey.

\section{Secondary analysis: effects of intervention on mindfulness}

Mean MAAS scores for each group are shown in the online supplemental table. Mindfulness scores at T1 were slightly higher 
Table 2 Demographics of non-completers at post-treatment and at 3 months follow-up

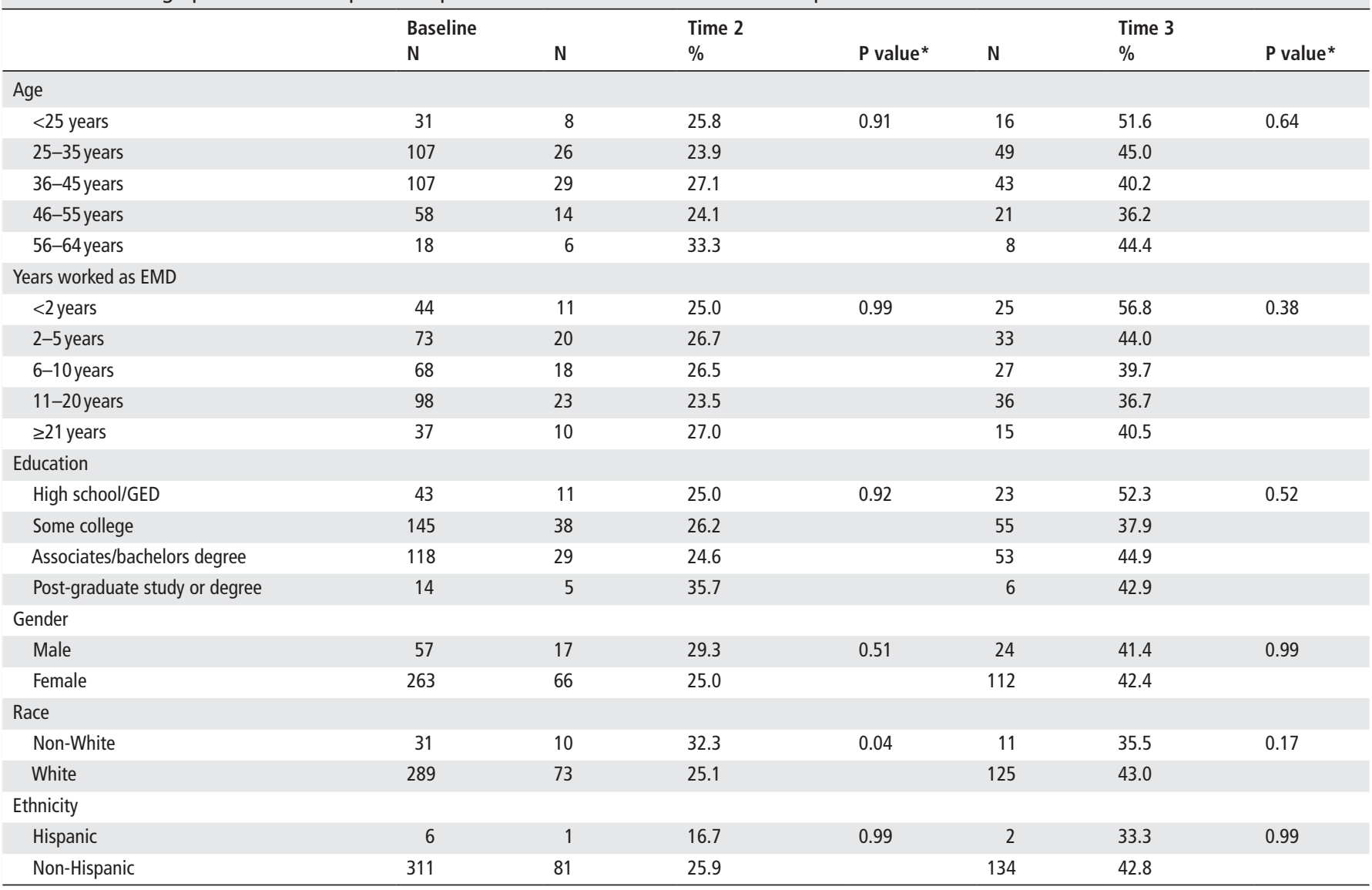

*Indicates $p$ value associated with difference from baseline.

EMD, emergency medical dispatchers.

in the intervention group than in the control group $(p=0.029)$. No statistically significant differences were observed in change in mindfulness between the intervention and control groups at T2 or T3.

At baseline, participants who had higher MAAS scores had lower SOSI scores $(\mathrm{r}=-0.71, \mathrm{p}<0.001)$. Pre-post change in MAAS score was associated with change in SOSI scores, with participants with greater increases in MAAS scores having, on average, greater decreases in SOSI scores $(\mathrm{r}=-0.53, \mathrm{p}<0.001)$. Control participants with higher MAAS scores at baseline showed greater decreases in MAAS between T1 and T2. To allow for the possibility that higher MAAS scores at $\mathrm{T} 1$ in the intervention group reduced the apparent effectiveness of the intervention, we conducted an analysis of the intervention change in MAAS scores from T1 to T2, controlling for T1 score. This analysis did not show any statistically significant effects of intervention on change in MAAS score or in the slope of the association between $\mathrm{T} 1$ and $\mathrm{T} 2$.

\section{DISCUSSION}

This study examined the efficacy of an online MBI tailored for EMDs to reduce stress. Results documented significant reductions in stress scores among EMDs randomised to the intervention group, with pre-post effect sizes in the small to moderate range (Cohen's $\mathrm{d}=0.34$ ). Results at 3 months follow-up indicated that intervention participants continued to show reduced stress scores when compared with baseline, and in comparison, to control participants (Cohen's $d=0.22$ ). Although study attrition was greater among control participants, sensitivity analyses revealed that study results are robust to the impact of bias introduced by differential attrition across groups.

Table 3 Change in C-SOSI scores from baseline between the control and intervention groups

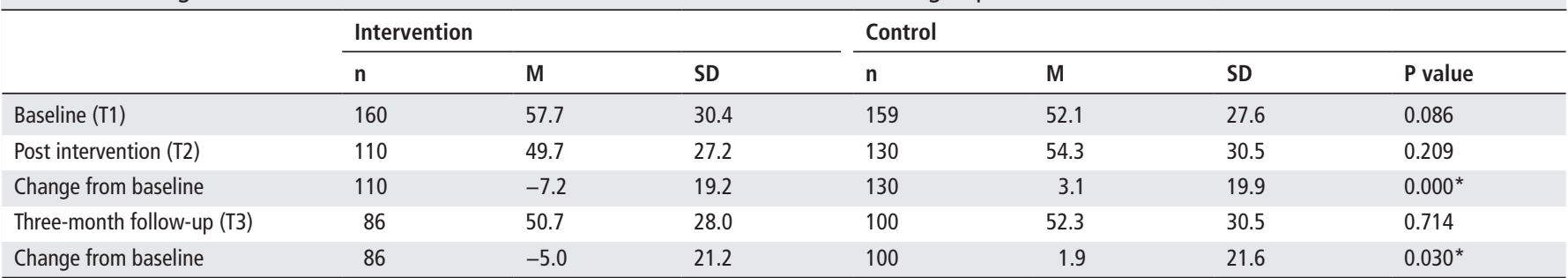

*Statistically significant at the $\alpha=0.05$ level.

C-SOSI, Calgary Symptoms of Stress Inventory. 
Table 4 C-SOSI scores between intervention participants who completed the majority of the lessons (at least 6 out of 7) and participants in the control group who completed a majority of the lessons (at least 6 out of 7) when offered the training at the end of the study

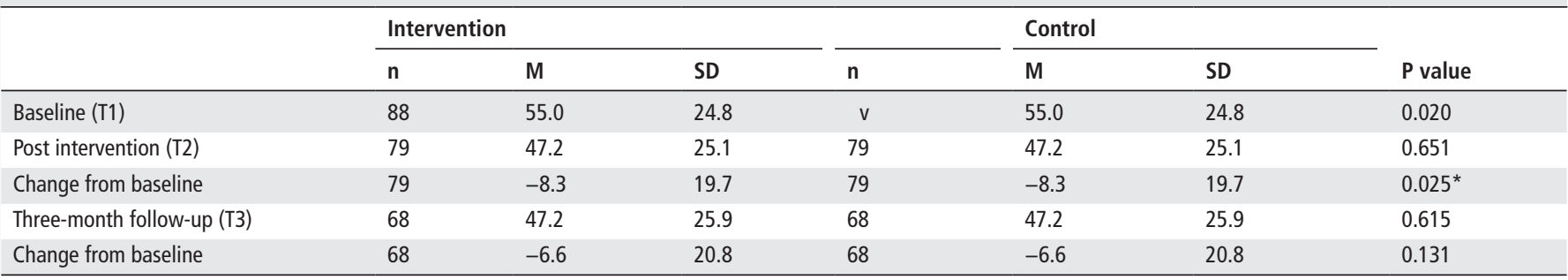

*Statistically significant at the $\alpha=0.05$ level.

C-SOSI, Calgary Symptoms of Stress Inventory.

\section{CONCLUSIONS}

Previous research testing MBIs among police officers ${ }^{14} 15$ were limited to within-subject designs. The results of this randomised controlled trial support the benefits of a tailored online MBI targeting stress in EMDs. The effect sizes observed were smaller than those reported in a recently published meta-analysis of MBIs targeting workplace stress. ${ }^{12}$ It is possible that the online format reduced the overall impact of the intervention. Though an in-person approach may have produced greater impact, consultants from within 9-1-1 agencies reported significant logistic challenges to implementing in-person group trainings. As an added advantage, the online format creates greater geographical reach and reduces costs associated with in-person approaches.

Enhanced mindfulness scores were not observed following the training. It is possible that a person begins to recognise low levels of mindfulness, and high levels of mindlessness, on learning about and practising mindful activities. Further, recent meta-analyses reveal that MBIs in the workplace tend to have only small ${ }^{25}$ to medium effect sizes in enhancing mindfulness, ${ }^{26}$ and a recent meta-analysis showed that clinical outcomes show significantly greater change following MBIs than self-reported mindfulness. ${ }^{27}$ Though we examined mindfulness as a possible mechanism of change, researchers have shown enhanced insight, acceptance, attention regulation and theoretical transparency, ${ }^{28} 29$ as well as increased compassion, increased meta-awareness and reduced worry $^{29}$ as potentially potent mechanisms of MBI-related change. These factors were not measured in the current study to reduce participant burden, but may have better accounted for the reductions in stress seen among the intervention group. Despite the non-significant findings in regard to mindfulness scores, greater positive change in mindfulness scores was associated with greater reductions in stress post training and at follow-up. These results suggest that while mean levels of mindfulness did not differ as a function of exposure to the intervention, greater improvement in mindfulness could be at least partially responsible for reductions in stress symptom scores among intervention participants.

\section{Strengths and limitations}

Although the majority of the intervention group completed 6 or 7 of the training modules $(55.3 \%)$, the average number of days per week during which exercises were practised was 2 (ranging from 0 to 7 ). Qualitative data provided by participants indicated that a barrier to engagement with intervention materials was inability to complete the intervention at work. Although enroled call centres initially indicated willingness to allow participants time during work hours to complete the intervention, many participants were not provided this opportunity. Work within the EMD industry is typically marked by lengthy shifts, mandatory overtime and shift work scheduling, which may have made it challenging to schedule a specific time to engage with the exercises (which aids in the development of any habit) or allocate time to engage with the intervention materials. Future effectiveness studies are needed to identify and remove barriers to engagement. Future trials may also identify the minimum level of engagement necessary to promote change so that the number of modules, or length of modules, may be reduced. Despite these limitations, the intervention appears to have reduced stress for those who engaged with it, even if engagement was limited.

Relatedly, a significant minority of individuals did not complete the intervention and/or post-intervention survey. Attempts were made to contact non-completers to clarify reasons behind their attrition. In these cases, many emails were returned as invalid, perhaps indicating that these individuals had left employment at their centre. The EMD industry suffers from a high annual turnover rate. In one study of 9-1-1 telecommunicators, $10 \%$ of participants left the industry in just 1 year, with the $10 \%$ estimate accounting for only confirmed cases of job attrition, which was likely an underestimate of true job attrition. ${ }^{30}$ Follow-up with centre directors to determine whether participants had left the industry could have clarified the nature of participants' attrition. Without a valid email address, participants could not be contacted to identify factors contributing to their attrition. Though attrition was higher than desired, the average rate of attrition at post-treatment in this sample $(24.7 \%)$ is consistent with the rate of attrition identified in meta-analyses for in-person MBIs (0\%-56\%) ${ }^{12}$ and online MBIs $(0 \%-60 \%) .{ }^{13}$ Study attrition also differed significantly across groups. Sensitivity analyses revealed that much larger changes in stress score between completers and non-completers would have been needed to significantly change study results. Yet, future research could benefit from identifying ways to retain control participants who may feel less compelled to complete surveys in the absence of direct benefits.

As an additional limitation, the MAAS has been critiqued as a measure of mindfulness, with some arguing that the unidimensional measure fails to capture important components of mindfulness such as acceptance and non-judgemental awareness. ${ }^{31}$ Future research may benefit from the incorporation of a different mindfulness measure such as the Five Facet Mindfulness Questionnaire. ${ }^{32}$ It is also possible that reductions in stress post intervention allowed for enhanced opportunity to practise mindful awareness. The direction of effects in this study must therefore be considered speculative. Finally, participants were not asked to specify the exercises they completed as part of their daily practice. As such, it cannot be determined whether specific exercises (ie, mindful eating and mindful movement) or practices (ie, guided meditation) were completed more frequently or were most impactful. 


\section{FUTURE RESEARCH}

This study provided initial evidence of the efficacy of 9-1-1 Destress. Future directions may include a focus on the impacts of different intervention dosages and use of the training in preventive efforts. A recent meta-analysis examining the impact of online MBIs in preventive medicine found moderate to large effects in perceived stress among non-clinical samples of volunteers. ${ }^{33}$ Identification of the modules of the training that were more impactful may also assist in developing more intensive materials in content areas found to be most beneficial. As stress levels were measured only pre and post intervention, changes in stress in response to specific modules cannot be determined in this study and should be investigated in future research. As a first responder worker population at elevated risk of adverse psychological outcomes, such as post-traumatic stress disorder and depression, ${ }^{34}$ examination of whether 9-1-1 Destress can deter the onset or progression of clinical phenomena is warranted. Prior research has also shown that MBIs can improve overall functioning in other areas important for EMDs. For example, MBIs have been shown to reduce stress-related working memory impairments in students, to enhance occupational functioning in working adults experiencing anxiety and to increase resilience and decrease burnout in police officers. ${ }^{35-37}$ Further exploration of the effects of this training on such outcomes in EMDs could shed light on the beneficial impacts of MBIs for our very first responders.

Correction notice This article has been corrected since it published Online First.

Contributors The research project was originally conceptualised by HM, RC and $\mathrm{ML}$. The intervention was developed by RC and ML. The design of the study was co-developed by all study authors. ML drafted the original manuscript with the exception of the results and tables, which were initially drafted by IP. IP completed all statistical analyses. SS was instrumental in conducting the research and provided feedback on the manuscript draft. RB, DR, JB and HM provided iterative feedback on the manuscript.

Funding This project was supported by grant number ROH010536 funded by the National Institute for Occupational Safety and Health (NIOSH), the Centers for Disease Control and Prevention. The contents are solely the responsibility of the authors and do not necessarily represent the official views of the Centers for Disease Control and Prevention or the Department of Health and Human Services.

Competing interests None declared.

Patient consent for publication Not required.

Provenance and peer review Not commissioned; externally peer reviewed.

\section{REFERENCES}

1 Larsson G, Berglund AK, Ohlsson A, et al. Daily hassles, their antecedents and outcomes among professional first responders: A systematic literature review. Scand J Psychol 2016;57:359-67.

2 Violanti JM. Introduction to special issue police stress and trauma: recent perspectives. Int J Emerg Ment Health 2013;15:213-5.

3 Chiu S, Webber MP, Zeig-Owens R, et al. Validation of the center for epidemiologic studies depression scale in screening for major depressive disorder among retired firefighters exposed to the World Trade Center disaster. J Affect Disord 2010;121:212-9.

4 Hartley TA, Violanti JM, Fekedulegn D, et al. Associations between major life events, traumatic incidents, and depression among Buffalo police officers. Int J Emerg Ment Health 2007:9:25-35.

5 Hartley TA, Burchfiel CM, Fekedulegn D, et al. Health disparities in police officers: comparisons to the U.S. general population. Int J Emerg Ment Health 2011;13:211-20.

6 Poston WS, Haddock CK, Jahnke SA, et al. The prevalence of overweight, obesity, and substandard fitness in a population-based firefighter cohort. J Occup Environ Med 2011:53:266-73.

7 Meischke H, Painter I, Lilly MM, et al. An exploration of sources, symptoms and buffers of occupational stress in 9-1-1 emergency call centers. Ann Emerg Dispatch Response 2015;3:28-35.
8 Kabat-Zinn J, Lipworth L, Burney R. The clinical use of mindfulness meditation for the self-regulation of chronic pain. J Behav Med 1985;8:163-90.

9 Dixon HC, Overall NC. Dispositional mindfulness attenuates the link between daily stress and depressed mood. J Soc Clin Psychol 2016;35:255-68.

10 Bergin AJ, Pakenham KI. The stress-buffering role of mindfulness in the relationship between perceived stress and psychological adjustment. Mindfulness 2016;7:928-39.

11 WILLIAMS V, Ciarrochi J, Deane FP. On being mindful, emotionally aware, and more resilient: Longitudinal pilot study of police recruits. Aust Psychol 2010;45:274-82.

12 Virgili M. Mindfulness-based interventions reduce psychological distress in working adults: a meta-analysis of intervention studies. Mindfulness 2015;6:326-37.

13 Huang SL, Li RH, Huang FY, et al. The potential for mindfulness-based intervention in workplace mental health promotion: results of a Randomized Controlled Trial. PLoS One 2015;10:10

14 Christopher MS, Goerling RJ, Rogers BS, et al. A pilot study evaluating the effectiveness of a mindfulness-based intervention on cortisol awakening response and health outcomes among law enforcement officers. J Police Crim Psychol 2015.

15 Bergman AL, Christopher MS, Bowen S. Changes in facets of mindfulness predict stress and anger outcomes for police officers. Mindfulness 2016;7:851-8.

16 Spijkerman MP, Pots WT, Bohlmeijer ET. Effectiveness of online mindfulness-based interventions in improving mental health: A review and meta-analysis of randomised controlled trials. Clin Psychol Rev 2016;45:102-14.

17 Carlson LE, Thomas BC. Development of the Calgary Symptoms of Stress Inventory (C-SOSI). Int J Behav Med 2007;14:249-56.

18 Eklund C, Elfström ML, Eriksson Y, et al. Development of the web application My Stress Control_-Integrating theories and existing evidence. Cogent Psychol 2018;5:5.

19 Brown KW, Ryan RM. The benefits of being present: mindfulness and its role in psychological well-being. J Pers Soc Psychol 2003;84:822-48.

20 Kersemaekers W, Rupprecht S, Wittmann M, et al. A workplace mindfulness intervention may be associated with improved psychological well-being and productivity. A preliminary field study in a company setting. Front Psychol 2018:9.

21 Pascal A, Dauvier B, Congard A. Individual differences in the effects of a positive psychology intervention: Applied psychology. Pers Individ Dif 2018;122:140-7.

22 Meischke $H$, Lilly $M$, Beaton $\mathrm{R}$, et al. Protocol: a multi-level intervention program to reduce stress in 9-1-1 telecommunicators. BMC Public Health 2018;18:18.

23 Kabat-Zinn J. Full catastrophe living: using the wisdom of your body and mind to face stress, pain, and illness. New York: Dell Publishing.

24 R Core Team. R: A language and environment for statistical computing. R Foundation for Statistical Computing, Vienna, Austria. 2016 https://www.R-project.org/.

25 Lomas T, Medina JC, Ivtzan I, et al. A systematic review and meta-analysis of the impact of mindfulness-based interventions on the well-being of healthcare professionals. Mindfulness 2018;30

26 Bartlett L, Martin A, Neil AL, et al. A systematic review and meta-analysis of workplace mindfulness training randomized controlled trials. J Occup Health Psychol 2019:24:108-26.

27 Goldberg SB, Tucker RP, Greene PA, et al. What can we learn from randomized clinical trials about the construct validity of self-report measures of mindfulness? A metaanalysis. Mindfulness 2018.

28 Grabovac AD, Lau MA, Willett BR. Mechanisms of mindfulness: a buddhist psychological model. Mindfulness 2011;2:154-66.

29 van der Velden AM, Kuyken W, Wattar U, et al. A systematic review of mechanisms of change in mindfulness-based cognitive therapy in the treatment of recurrent major depressive disorder. Clin Psychol Rev 2015;37:26-39.

30 Meischke H, Painter IS, Stangenes SR, et al. Simulation training to improve 9-1-1 dispatcher identification of cardiac arrest: A randomized controlled trial. Resuscitation 2017;119:21-6.

31 Sauer S, Walach $\mathrm{H}$, Schmidt S, et al. Assessment of mindfulness: review on state of the art. Mindfulness 2013;4:3-17.

32 Baer RA, Smith GT, Hopkins J, et al. Using self-report assessment methods to explore facets of mindfulness. Assessment 2006;13:2745-

33 Jayewardene WP, Lohrmann DK, Erbe RG, et al. Effects of preventive online mindfulness interventions on stress and mindfulness: A meta-analysis of randomized controlled trials. Prev Med Rep 2017:5:150-9.

34 Lilly MM, Allen CE. Psychological Inflexibility and Psychopathology in 9-1-1 Telecommunicators. J Trauma Stress 2015:28:262-6.

35 Banks JB, Welhaf MS, Srour A. The protective effects of brief mindfulness meditation training. Conscious Cogn 2015;33:277-85.

36 Hoge EA, Guidos BM, Mete M, et al. Effects of mindfulness meditation on occupational functioning and health care utilization in individuals with anxiety. J Psychosom Res 2017:95:7-11.

37 Kaplan JB, Bergman AL, Christopher $M$, et al. Role of resilience in mindfulness training for first responders. Mindfulness 2017:8:1373-80. 